\title{
COMA E SÍNDROME DE HERNIAÇÃO TRANSTENTORIAL CONSEQUENTES A LESÃO HEMISFÉRICA NÃO EXPANSIVA AGUDA
}

\author{
RICARDO DE OLIVEIRA-SOUZA", MARCOS BENCHIMOL **
}

RESUMO - O presente trabalho retrata o caso de uma paciente que entrou em coma por infarto extenso do hemisfério cerebral direito e desenvolveu sinais clínicos característicos do estágio diencefálico da hemiação transtentorial central. A TC, todavia, não evidenciou os efeitos classicamente descritos de compressão ou distorção da transiçāo mesodiencefálica, mas duas imagens hipodensas, de idades diferentes, alojadas, repectivamente, nos territórios superficial e profundo das artérias cerebral média e anterior e no lobo frontal direito. Concordando com a piora clínica ao longo dos seis dias que antecederam a morte, a última TC revelou as imagens esperadas de deslocamentos do encéfalo. $O$ caso se assemelha a outros da literatura, $\mathrm{em}$ testemunho de que os sinais clínicos de efeitos de massa no interior do crânio podem ser reproduzidos por comprometimento funcional agudo mono-hemisférico na ausência de deslocamentos físicos da massa encefálica. $O$ papel desempenhado por distorções encefálicas na gênese e no prognóstico de curto-prazo do coma permanece em aberto.

PALAVRAS-CHAVE: coma, herniaçāo transtentorial, sistema reticular ativador ascendente, diásquise.

Coma and central herniation syndrome due to an acute non-expanding hemispheral mass

SUMMARY - An 81-year-old woman developed left hemiplegia and coma due to a right hemisphere infarct and died 6 days later. When first seen in coma she had the classical signs of descending central herniation in the diencephalic stage. The CT scan of the third day showed a large hypodense area occupying the superficial and deep territories of the middle and anterior cerebral arteries on the left as well as a frontal cortico-subcortical hypodense area indicative of an older infarct on the opposite hemisphere. No mass effects were apparent. She followed a downhill course with signs of brainstem deterioration. A second CT scan a few hours before death revealed the expected pattem of brain shift and herniations. This case adds to the available evidence showing that the clinical signs of encroachment of supratentorial structures upon the basal forebrain can be reproduced by an acute mono-hemispheral lesion without visible mass effects. It indicates, further, that the role of intracranial displacements in the genesis and short-term prognosis of coma remains an unsettled matter. In at least a few number of cases, diaschisis might play a major role.

KEY WORDS: coma, transtentorial herniation, ascending reticular activating system, diaschisis

O estupor e o coma das lesões supratentoriais unilaterais agudas são consensualmente justificados pela compressão do tronco encefálico pela herniação do uncus ou do diencéfalo através da incisura da tenda do cerebelo ${ }^{8}$. Esse mecanismo, há muito reconhecida ${ }^{18}$, foi formalmente explicitado na monografia de Plum e Posner ${ }^{9}$, que se basearam em verificaçōes post mortem de deslocamentos do encéfalo entre os diferentes compartimentos intracranianos facilitados por gradientes verticais ou diagonais ("cones de pressão") ocasionados por massas expansivas agudas. Com o aprimoramento das técnicas de imagem, todavia, a ausência de herniações cerebrais em pacientes em coma por lesōes hemisféricas agudas foi sendo cada vez mais observada. Fishert estudou 20 pacientes com doença hemisférica unilateral aguda e sinais clínicos convencionais de herniaçāo

Serviço de Clínica Médica C (Prof. Dr. Omar da Rosa Santos). Hospital Universitário Gaffrée e Guinle (UNI-RIO), Rio de Janeiro, RJ;* Professor Assistente; "Professor Auxiliar. Aceite: 15-agosto-1995. 
tentorial. Em nenhum deles a calcificação da pineal se achava abaixo do plano da calcificação do plexo coróide contralateral, mas desviada horizontalmente em relação à linha média. Os efeitos compressivos sobre o tronco foram tratados como "epifenômeno tardio e irreversível", sem maior importância para a produção de estupor e coma, que precederiam a herniação. Ropper ${ }^{10}$ estudou 24 pacientes com massas intracranianas agudas lateralizadas, correlacionando a distância da pineal da linha média com o nível de vigília e explicando a reduçāo inicial e persistente da vigília pelo deslocamento horizontal da porção do tronco (junção mesodiencefálica) que atravessa a tenda do cerebelo. Essas observações foram reproduzidas em estudos com ressonância nuclear magnética (RNM) ${ }^{3,11}$ comparando os deslocamentos horizontais e verticais do diencéfalo no plano coronal, confirmando, aparentemente, a exigência de acometimento bi-hemisférico para interferência no nível de consciência ("coma bi-hemisférico"). Além disso, ajustavam-se de maneira singular às concepções modernas sobre os sistemas de ativação do cérebro, cujas fibras confluem nas cercanias da transição mesodiencefálica ${ }^{16}$. A partir dessas verificações, o deslocamento da pineal passou a sinalizar deformação proporcional dos axônios ativadores ascendentes de cada lado da linha média, compatível, portanto, com as diminuições do nível de consciência clinicamente aparentes. Da perspectiva atual, acima sumariada, não parece haver lugar para o coma secundário a lesão aguda de um hemisfério sem efeitos de massa associados ("coma mono-hemisférico"). Não obstante, evidências em contrário permeiam a literatura dos últimos anos ${ }^{15}$. A série mais numerosa dedicada ao efeito das lesões cerebrais unilaterais sobre o nível de consciência foi publicada por Salazar e col. ${ }^{15}$, que reuniram 342 veteranos de guerra com lesōes penetrantes do crânio por projéteis de alta velocidade. Cincoenta e três (15\%) entraram em coma no momento do impacto e assim permaneceram por horas a dias. As perdas de massa do hemisfério esquerdo produziram reduçāo da vigília com mais frequência que as do hemisfério direito que, quando o fizeram, foram consideravelmente maiores. Embora não mencionado, os autores implicaram a possibilidade da ocorrência de estupor e coma na ausência de distorções da massa encefálica, atribuindo ao hemisfério esquerdo papel dominante na gênese da vigília.

O presente trabalho nasceu da observação de uma paciente acometida de hemiplegia esquerda e declínio acelerado do nível de vigília. Como a tomografia computadorizada (TC) da internação não evidenciava efeitos de massa, perguntamo-nos por que, afinal, a paciente entrara em coma. Tais circunstâncias se opunham às idéias costumeiras sobre os mecanismos do coma estrutural ${ }^{8}$, levandonos a reconsiderar suas bases fisiopatológicas.

\section{RELATO DO CASO}

Uma senhora de 81 anos de idade (CJC), destra, viveu independente e ativa até março-1995, quando apresentou deficiência motora no dimídio esquerdo (E) em grau suficiente para prejudicar a locomoção. $\mathrm{Na}$ noite do dia seguinte, embora acamada, mantinha-se acordada e reconhecia os familiares pelo nome, errando a orientação temporal (data e dia da semana) por 1 dia. Internada no terceiro dia (d3) por sonolência crescente e incontinência dos esfincteres, mostrava-se, ao exame, irresponsiva, mandíbula hipotônica e respiração de CheyneStokes, sem sinais meníngeos, olhos fechados e desviados para a direita, sem piscar, pupilas isocóricas, pequenas e fotorreagentes. Arreflexia cómeo-palpebral E aferente. Sob induçāo nociceptiva (Marie-Foix ${ }^{7}$ ), elevava o braço D sem localizar o estímulo. O reflexo vestíbulo-ocular (RVO), com água fria, induzia desvio conjugado dos olhos para o lado estimulado; o reflexo óculo-cefálico (ROC) produzia desvio conjugado dos olhos para o lado oposto ao da rotação da cabeça, sem sinais de oftalmoplegia internuclear (OIN). Reflexo plantar em extensão à E, indiferente a D. A TC do çânio, obtida no momento do exame neurológico, revelou as alterações encefálicas próprias da idade e extensa região hipodensa no hemisfério D, compatível com infarto. No hemisfério esquerdo, regiāo hipodensa no lobo frontal e alargamento do como ventricular adjacente, sugestiva de infarto antigo (Fig 1). A natureza da doença arterial de base não foi estabelecida. Nāo havia alteraçōes metabólicas ou infecciosas que justificassem o coma. Estavam normais, em particular, os seguintes resultados: hemograma, VHS, glicemia, uréia e creatinina, tempo e atividade da protrombina, TSH e hormônios tireoidianos, ionograma (sódio, potássio, cálcio, fósforo cloretos e magnésio), proteinograma, provas funcionais hepáticas e exame de urina. Progrediu com sinais de comprometimento descendente do tronco encefálico (Fig 2). No quinto dia de hemiplegia (d5), apresentava-se em atonia muscular generalizada, olhos fechados e divergentes, pupilas anisocóricas (D>E) e fixas à estimulação luminosa, reflexos córneo-palpebral e plantar abolidos, OIN bilateral (RVO e ROC). 


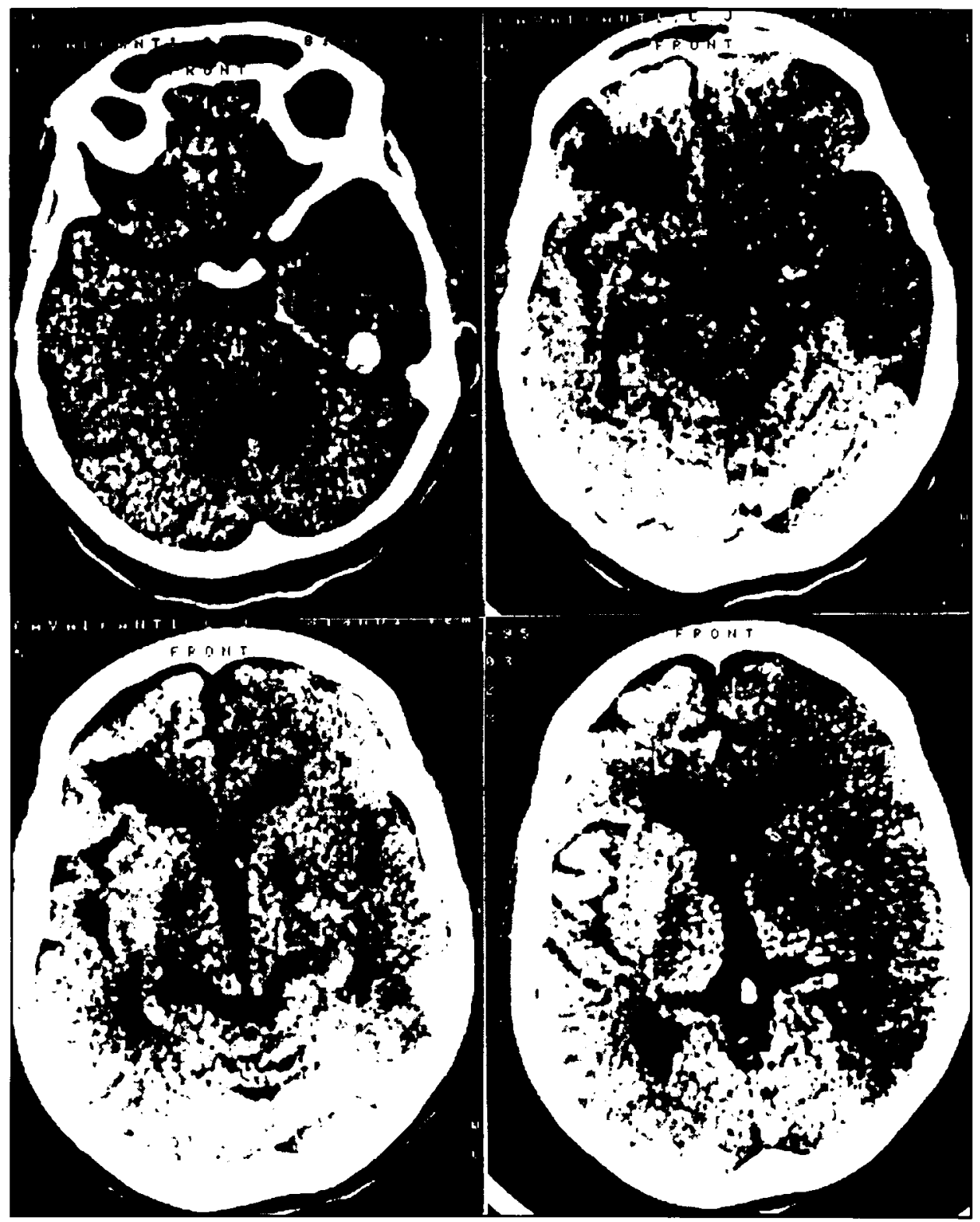

Fig l. TC de crânio (Somatom CRX, matriz 256x256, cortes paralelos à linha óbito-meatal, 8 mm) concomitante aos sinais clínicos de herniaçäo transtentorial central. A: (no alto, à esquerda) aumento dos sulcos corticais e dos ventrículos laterais, dilatação dos terceiro e quarto ventrículos, centrados; à direita, área hipodensa nos territórios superficiais e profundos das artérias cerebrais anterior e média; porção temporal dos ventrículos laterais simétrica, cisternas do ângulo ponto-cerebelar e pré-pontina visíveis; o uncus, de cada lado, näo oblitera as cisternas vizinhas. B: (no alto, à direita) cisternas peri-mesencefálicas visíveis e simétricas. $C$ : (em baixo, à esquerda) porçāo frontal dos ventrículos laterais e calcificação dos plexos coróides simétricas, cisterna quadrigeminal livre. D: (em baixo, à direita) preservação aparente dos territórios vértrebro-basilares, pineal centrada, apagamento da cisterna sylviana direita. 


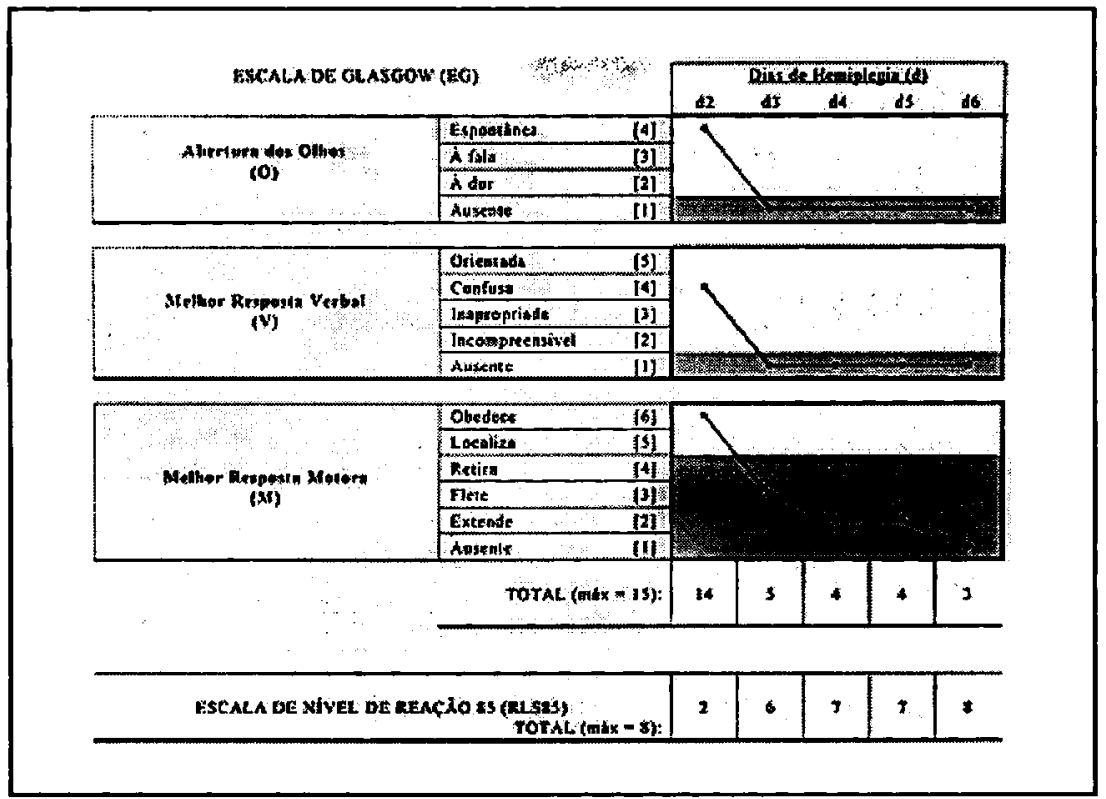

Fig 2. Representação gráfica da progressão neurológica a partir do segundo dia de hemiplegia

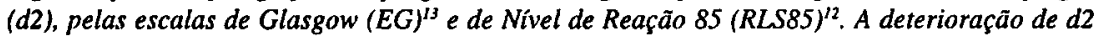
para $d 3$ correspondeu à transposição do "limiar do coma" (regiōes em cinza em EG) nas duas escalas $^{6,13,16}$. A TC da Fig 1 corresponde a d3, quando $E G=5(01 / \mathrm{NI} / \mathrm{M} 3)$ e RLS85 $=6$. Em d5, sinais clínicos de acometimento do tronco encefálico. A TC da Fig 3 foi realizada em d6 (OI/NII $M ! ; R L S 85=8$ ).

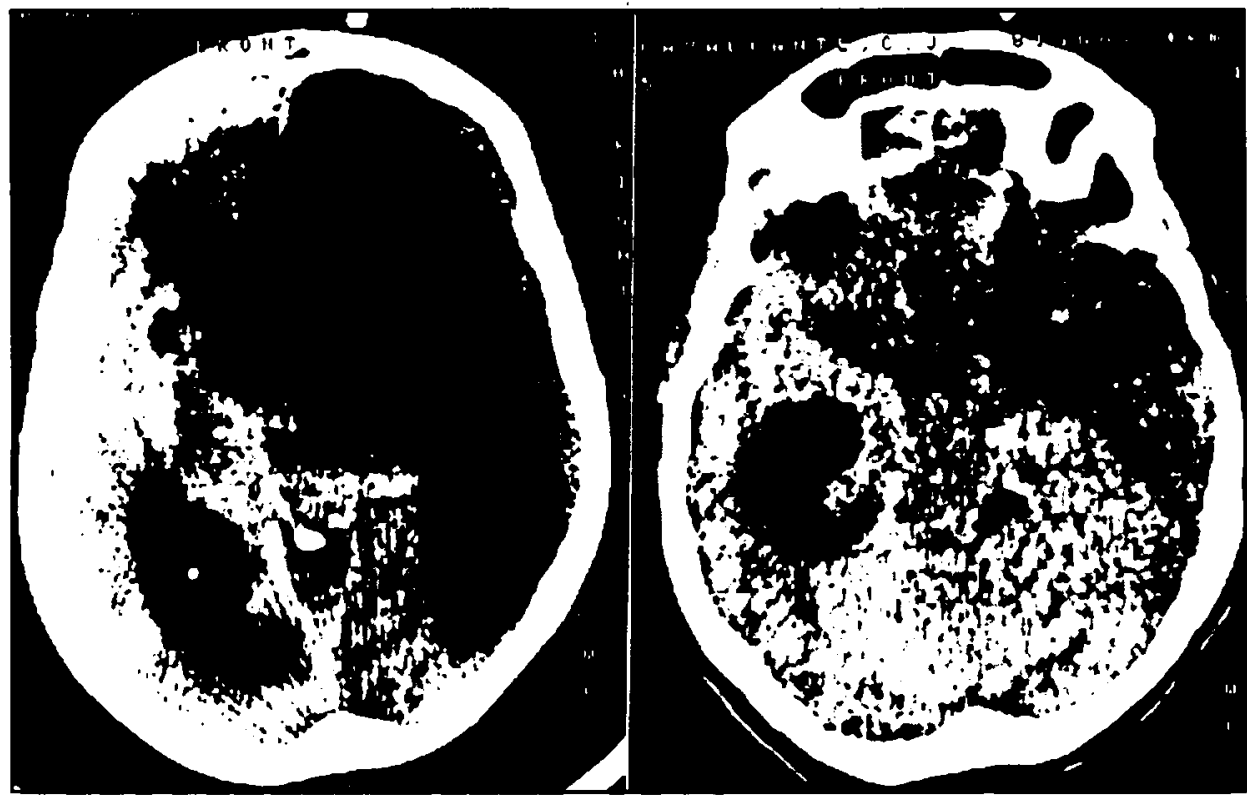

Fig 3. TC do dia anterior ao óbito (d6). Herniação transtentorial: expansão da lesão, desvio da linha média, colapso do ventrículo lateral, hérnia do uncus, dilataf̧āo do ventrículo lateral contralateral (hidrocefalia obstrutiva aguda) e apagamento das cisternas peri-mesencefálicas. 
No dia seguinte, deixou de reagir à estimulação nociceptiva, os reflexos comeanos e plantares desapareceram e as pupilas tornaram-se isocóricas e fixas. A TC de acompanhamento (d6) revelou imagem típica de herniação transtentorial (Fig 3). Faleceu horas depois.

\section{COMENTÁRIOS}

O aspecto central do caso diz respeito à ocorrência de coma por lesão cerebral unilateral, na ausência de efeitos de massa. Inicialmente, a paciente preencheu os requisitos clínicos - (a) respiratórios, (b) pupilares, (c) resposta à estimulação nociceptiva e (d) oculomotores - para 0 diagnóstico do estágio diencefálico da herniação transtentorial central ${ }^{9}$. Tal localização, no entanto, foi refutada pela TC, que não revelou a imagem prevista de deslocamento da base do hemisfério afetado, quer descendente, quer lateral ${ }^{19}$, surpreendendo nossas expectativas em três aspectos: (i) ausência de evidência anatômica de herniação central, (ii) ausência de deslocamento horizontal da transiçāo mesodiencefálica e (iii) pelo fato de a lesão ter-se alojado no hemisfério direito, considerado não dominante para a vignlia. Os achados tomográficos mostraram-se compatíveis com infarto do território carotídeo direito, desenvolvido sobre um cérebro senescente difusamente atrofiado, que abrigava outro infarto, mais antigo, no lobo frontal esquerdo.

Como explicar, então, a precedência temporal do coma em relação aos sinais radiológicos de deslocamentos do encéfalo?

A possibilidade mais simples se refere à presença de lesões no tronco ou na base do cérebro, não detectadas pela TC, alojadas em pontos críticos de passagem ou origem do sistema reticular ativador ascendente $^{16}$. Na ausência de confirmação por RNM ou por anatomia patológica, esta possibilidade não pode ser refutada. Todavia, a mesma limitação se aplicaria aos estudos que empregaram TC como instrumento de visualização direta do encéfalo ${ }^{10.15}$. Outra possibilidade diz respeito à adição do infarto recente ao infarto frontal contralateral, antigo, ambos destruindo parcela majoritária das fibras ativadoras ascendentes, originárias do prosencéfalo basal e terço rostral do tegmento ${ }^{16}$. Fenômeno idêntico está bem documentado no sistema motor de pacientes que desenvolveram hemiplegias capsulares bilaterais sequenciais ${ }^{5} \mathrm{e}$ em animais submetidos à destruiçāo do tegmento em tempos cirúrgicos distintos ${ }^{1}$. Finalmente, uma terceira alternativa diz respeito ao coma mono-hemisférico e, ao contrário das anteriores, privilegia a operação de mecanismos funcionais em oposição aos mecânicos (compressões, deslocamen.os) e anatômicos (lesão bilateral do sistema ativador). Salazar e col. ${ }^{15}$ reviram as principais publicaçōes sobre o coma por compromətimento de um hemisfério por causas que variam das afeç̧ões cerebrovasculares à depressão química do teste de Wada. Embora alojada no hemisfério não dominante para a vigília, lesões comparáveis mostraramse volumosas o bastante para produzir coma ${ }^{15}$. Um dos mecanismos pelos quais uma lesão cerebral unilateral poderia levar ao coma é o da "diásquise transcalosa", expressão utilizada para descrever a depressäo funcional de um hemisfério ocasionada pelo comprometimento estrutural do outro ${ }^{2}$. Esta hipótese prediz que a lesão aguda isquêmica de um hemisfério produzirá estupor e coma por indução - via corpo caloso - de diásquise no hemisfério oposto, que, somada aos efeitos destrutivos do infarto, resultará no estado coma. A participação da diásquise transcalosa no coma não foi estudada sistematicamente. Não obstante, representa a hipótese mais parcimoniosa, uma vez que concilia o requisito de comprometimento bi-hemisférico com a presença de lesāo em apenas um.

Sejam quais forem as explicações, a reprodução desses achados poderia influenciar algumas das condutas e concepçōes em voga, como, por exemplo, a necessidade de obtenção da primeira TC o mais cedo possível. Se ficasse demonstrado que, ao menos em determinados pacientes, o coma nāo se deve a efeitos mecânicos, a indicação de cirurgias descompressivas de urgência exigiria revisão. Sendo a diásquise dependente da operaçāo de mecanismos funcionais, vale dizer químicos, a demonstraçāo de sua participação na gênese do coma deslocaria a ênfase terapêutica para o desenvolvimento de manipulações neurofarmacológicas específicas. Aplicações bem sucedidas desse conceito são, há muito, reconhecidas ${ }^{12}$. A noção prevalente de que a previsāo de recuperação da 
consciência depende da ocorrência de hérnias transtentoriais pode ser verdadeira em certos casos. Em outros, o prognóstico seria determinado por fatores atuando em fases precoces da instalação da lesão, o que, obviamente, implicaria em novas atitudes terapêuticas e inspirações para a pesquisa clínica.

\section{REFERENCIAS}

1. Adametz JH. Rate of recovery of functioning in cats with rostral reticular lesions: an experimental study. J Neurosurg 1959, 16:85-98.

2. Feeney DM, Baron JC. Diaschisis. Stroke 1986, 17:817-830.

3. Feldmann E, Gandy SE, Becker R, Zimmerman R, Thaler HT, Posner JB, Plum F. MRI demonstrates descending transtentorial herniation. Neurology 1988, 38:697-701.

4. Fisher CM. Acute brain herniation; a revised concept. Semin Neurol 1984, 4:417-421.

5. Fisher CM. Concerning the mechanism of recovery in stroke hemiplegia. Can J Neurol Sci 1992, 19:57-63.

6. Jagger J, Jane JA, Rimel R. The Glasgow Coma Scale: to sum or not to sum? Lancet 1983, 2:97.

7. Marie P, Foix C. Le phénomène de la face. Rev Neurol (Paris) 1914-1915, 28:778-779.

8. Masson C, Cambier J, Masson M. Mécanisme physiopathologique des comas. Rev Pract (Paris) 1989, 39:2393-2396.

9. Plum F, Posner JB. The diagnosis of stupor and coma. Ed 3. Philadelphia. F.A.Davis, 1980, p 103-113.

10. Ropper AH. Lateral displacement of the brain and level of consciousness in patients with an acute hemispheral mass. N Engl J Med 1986, 314:953-958.

11. Ropper AH. A preliminary MRI study of the geometry of brain displacement and level of consciousness with acute intracranial mass. Neurology 1989, 39:622-627.

12. Ross ED, Stewart RM. Akinetic mutism from hypothalamic damage: successful recovery with dopamine agonists. Neurology, 1981, 31:1435-1439.

13. Rossitti S, Starmark J-E, Stålhammar D. Manual operacional da Escala de Nível Reativo (RLS85). Arq Neuropsiquiatr 1993, 51:103-106.

14. Sacco RL, VanGool R, Mohr JP, Hauser WA. Nontraumatic coma. Glasgow Coma Score and coma etiology as predictors of 2-week outcome. Arch Neurol 1990, 47:1181-1184.

15. Salazar A, Grafman JH, Vance SC, Weingartner H, Dillon JD, Ludlow C. Consciousness and amnesia after penetrating head injury: neurology and anatomy. Neurology 1986, 36:178-187.

16. Saper CB. Diffuse cortical projection systems: anatomical organization and role in cortical function. In Mount castle VB, Plum F (eds). Handbook of Physiology I, The Nervous System V, Higher Functions of the Brain. Bethesda: American Physiological Society, 1987 p169-210.

17. Starmark J-E, Lindgren S. Is it possible to define a general "conscious level"? Acta Neurochir (Wien), 1986, 36 (Suppl):103-105.

18. Vincent $C$, David $M$, Thiébaut $F$. Le cône de pression temporal dans les tumeurs des hémisphères cérébraux: sa symptomatologie, sa gravité; les traitements qu'il convient de lui opposer. Rev Neurol (Paris), 1936, 65:536-545.

19. Weisberg L, Nice C. Cerebral computed tomography. a text-atlas, Ed 3. Philadelphia: W.B.Saunders, 1989, p 249-253. 